\title{
Focus on learning and memory
}

In this special issue of Nature Neuroscience, we feature an assortment of reviews and perspectives that explore the topic of learning and memory.

earning new information and skills, storing this knowledge, and retrieving, modifying or forgetting these memories over time are critical for flexibly responding to a changing environment. How these processes occur has fascinated philosophers, psychologists, and neuroscientists for generations, and the question continues to inspire research encompassing diverse approaches. In this special issue, Nature Neuroscience presents a collection of reviews and perspectives that reflects the breadth and vibrancy of this field. Many of these pieces touch on topics that have animated decades of investigation, including the roles of synaptic plasticity, adult neurogenesis, neuromodulation, and sleep in learning and memory. Yet recently developed technologies continue to provide novel insights in these areas, leading to the updated views presented here.

Synaptic plasticity, such as long-term potentiation and depression, remains the prevailing cellular model for learning and memory. While many presume that these processes are engaged by learning and mediate lasting changes in behavior, this link has yet to be conclusively demonstrated in vivo. Humeau and Choquet (https://doi. org/10.1038/s41593-019-0480-6) outline the latest tools that can be used to visualize and manipulate synaptic activity and signaling in behaving animals, and they discuss further advances that are needed to help bridge this gap in our understanding.

Neuroscientists have also long been intrigued by the role that the formation of new neurons could play in memory formation and maintenance of new memories. Miller and Sahay (https://doi.org/10.1038/s41593019-0484-2) integrate recent research on adult hippocampal neurogenesis to present a model of how the maturation of adult-born dentate granule cells contributes to memory indexing and interference.

While the neural mechanisms underlying memory acquisition and consolidation are relatively well-described, less is known about how memories are retrieved.

Frankland, Josselyn, and Köhler (https://doi. org/10.1038/s41593-019-0493-1) discuss how recent approaches that enable the manipulation of memory-encoding neural ensembles (termed 'engrams') have informed our current understanding of retrieval. They highlight the ways in which retrieval success is influenced by retrieval cues and the congruence between encoding and retrieval states. They also discuss important open questions in the field.

External stimuli and internal states can affect various aspects of learning and memory, which is mediated in part by neuromodulatory systems. Likhtik and Johansen (https://doi.org/10.1038/s41593019-0503-3) detail how acetylcholine, noradrenaline, and dopamine systems participate in fear encoding and extinction. They discuss emergent themes, including how neuromodulation can act throughout the brain or in specifically targeted regions, how it can boost selected neural signals, and how it can tune oscillatory relationships between neural circuits.

The efficacy of memory storage is also influenced by sleep. Klinzing, Niethard, and Born (https://doi.org/10.1038/s41593-0190467-3) review evidence from rodent and human studies that implicates reactivation of memory ensembles (or 'replay'), synaptic scaling, and oscillations during sleep in memory consolidation. They also discuss recent findings that suggest that the thalamus coordinates these processes.
Effective learning requires us to identify critical information and ignore extraneous details, all of which varies depending on the task at hand. Yael Niv (https://doi.org/10.1038/s41593-019-04708) discusses computational and neural processes involved in the formation of such task representations, how factors such as attention and context affect these representations, and how we use task representations to make decisions.

The ability to issue appropriate outputs in response to neural activity is a critical brain function, and is often disrupted in injury and disease. Maryam Shanechi (https://doi. org/10.1038/s41593-019-0488-y) discusses how 'closed-loop' brain-machine interfaces (BMIs) have been used to monitor motor impulses and in turn control prosthetic or paralyzed limbs in order to restore function. Furthermore, she discusses how manipulation of BMI parameters can aid the study of learning. Finally, she explores how BMIs could be used in a similar vein to monitor and correct aberrant mood processes in psychiatric disorders.

By highlighting the topic of learning and memory, we honor its importance and centrality in neuroscience, while also celebrating the ways that other disciplines, including psychology, cellular and molecular biology, computer science, and engineering fuel insights in this area. We hope to continue to publish outstanding research in this area, particularly studies that resolve long-standing questions, that develop or leverage new methodologies, and that integrate multiple approaches.

Published online: 24 September 2019 https://doi.org/10.1038/s41593-019-0509-x 